\title{
Characterization of stromal tumor-infiltrating lymphocytes and genomic alterations in metastatic lobular breast cancer
}

François Richard ${ }^{1}$, Samira Majjaj ${ }^{2}$, David Venet ${ }^{2}$, Françoise Rothé ${ }^{2}$, Julien Pingitore ${ }^{2}$, Bram Boeckx ${ }^{3,4}$, Caterina Marchio ${ }^{5,6}$, Florian Clatot $^{7,8}$, François Bertucci ${ }^{9}$, Odette Mariani ${ }^{10}$, Christine Galant ${ }^{11}$, Gert van den Eynden ${ }^{12}$, Roberto Salgado ${ }^{12}$, Elia Biganzoli ${ }^{13}$, Diether Lambrechts ${ }^{3,4}$, Anne VincentSalomon $^{10}$, Giancarlo Pruneri ${ }^{14-16}$, Denis Larsimont ${ }^{17}$, Christos Sotiriou $^{2, *}$, Christine Desmedt ${ }^{1, *}$

1. Laboratory for Translational Breast Cancer Research, Department of Oncology, KU Leuven, Leuven, Belgium.

2. Breast Cancer Translational Research Laboratory, Université Libre de Bruxelles, Institut Jules Bordet, U-CRC, Brussels, Belgium.

3. VIB Center for Cancer Biology, Leuven, Belgium.

4. Laboratory of Translational Genetics, Department of Human Genetics, Leuven, Belgium.

5. Department of Medical Sciences, University of Turin, Turin, Italy

6. FPO-IRCCS Candiolo Cancer Institute, Candiolo, Italy

7. Centre Henri-Becquerel, Department of Medical Oncology, Rouen, France.

8. Rouen University Hospital, IRON/Inserm U1245, Rouen, France.

9. Predictive Oncology Laboratory, Institut Paoli-Calmettes, CRCM, INSERM U1068, CNRS UMR7258, Aix-Marseille Université, Marseille, France

10. Department of Pathology, Institut Curie, Paris Sciences Lettres Research University, Paris, France.

11. Department of Pathology, Cliniques Universitaires Saint Luc, Brussels, Belgium

12. Department of Pathology, Sint Augustinus, Wilrijk, Belgium

13. Unit of Medical Statistics, Biometry and Bioinformatics "Giulio A. Maccacaro", Department of Clinical Sciences and Community Health, University of Milan, Campus Cascina Rosa, Fondazione IRCCS Istituto Nazionale Tumori, Milan, Italy.

14. Division of Pathology, European Institute of Oncology, University of Milan, Milan, Italy

15. Division of Pathology, Fondazione IRCCS Istituto Nazionale dei Tumori, Milan, Italy 
16. School of Medicine, University of Milan, Milan, Italy.

17. Department of Pathology, Institut Jules Bordet, Brussels, Belgium

*These authors contributed equally.

Running title:

Genomic characterization of metastatic lobular breast cancer

\section{Corresponding authors:}

Christos Sotiriou

Breast Cancer Translational Research Laboratory (BCTL)

Institut Jules Bordet

125 Boulevard de Waterloo

1000 Brussels, Belgium

Phone: +325413428

Email: christos.sotiriou@bordet.be

Christine Desmedt

Laboratory for Translational Breast Cancer Research (LTBCR)

Department of Oncology

KU Leuven

Herestraat 49, box 818

1000 Brussels, Belgium

Email: christine.desmedt@kuleuven.be

\section{Keywords}

Lobular breast cancer, metastasis, genomics, tumor infiltrating lymphocytes, endocrine resistance, progression. 


\section{Conflict of interest statement}

The authors declare no potential conflicts of interest. 


\section{Statement of translational relevance}

Invasive lobular carcinoma (ILC) represents the second most common histological breast cancer subtype after invasive ductal carcinoma (IDC) and mostly expresses the estrogen receptor (ER). Several genomic alterations have been associated with endocrine resistance in ER-positive breast cancer. However, so far it was unknown whether these alterations are preexisting or acquired after endocrine treatment, and whether their frequencies differ between ILC and IDC metastases. Here, we present evidence of possibly acquired alterations associated with endocrine resistance during disease evolution in approximately one out of five ILC patients. Additionally, the genomic landscape of metastatic ILC does present some clinically-relevant differences with regard to metastatic IDC. While the present results still need to be confirmed in larger cohorts of patients with metastatic ILC, they support the need to characterize the metastatic disease, either using a metastatic biopsy or circulating tumor DNA (ctDNA), to refine treatment for these patients. 


\begin{abstract}

\section{Purpose}

Invasive lobular carcinoma (ILC) represents the second most common histological breast cancer subtype after invasive ductal carcinoma (IDC). While primary ILC has been extensively studied, metastatic ILC has been poorly characterized at the genomic and immune level.
\end{abstract}

\title{
Experimental design
}

We retrospectively assembled the multi-centric EuroILC series of matched primary and metastatic samples from 94 patients with estrogen receptor (ER)-positive ILC. Stromal tumor infiltrating lymphocytes (sTIL) were assessed by experienced pathologists. Targeted sequencing and low pass whole genome sequencing were conducted to detect mutations and copy number aberrations (CNAs). We compared the frequencies of the alterations in EuroILC with those from patients with ER-positive metastatic ILC $(\mathrm{n}=135)$ and IDC $(\mathrm{n}=563)$ from MSK-IMPACT.

\section{Results}

Low sTIL levels were observed in ILC metastases, with higher levels in the mixed non-classic histology. Considering ILC metastases from EuroILC and MSK-IMPACT, we observed that $>50 \%$ of tumors harbor genomic alterations that have previously been associated with endocrine resistance. A matched primary/metastasis comparison in EuroILC revealed mutations (AKT1, ARIDIA, ESRl, ERBB2 or NF1) and CNAs (PTEN or NF1 deletion, CYP19A1 amplification) associated with endocrine resistance that were private to the metastasis in $22 \%(7 / 32)$ and $19 \%(4 / 21)$ of patients, respectively. An increase in $C D H 1, E R B B 2, F O X A 1$ and $T B X 3$ mutations, in $C D H 1$ deletions and a decrease in TP53 mutations was observed in ILC as compared to IDC metastases.

\section{Conclusions}

ILC metastases harbor genomic alterations that may potentially explain endocrine resistance in a large proportion of patients, and present genomic differences as compared to IDC metastases. 


\section{Background}

Invasive lobular carcinoma (ILC) accounts for up to $15 \%$ of all invasive breast cancers and represents the second most common histological subtype of invasive breast cancer after invasive breast carcinoma of no special type, commonly referred to as invasive ductal carcinoma (IDC)(1). Lobular tumors are typically characterized by small discohesive cells that lack membranous Ecadherin expression. These tumors generally express the estrogen receptor (ER) and lack HER2 amplification. Patients with ILC tend to relapse later(2) and present different metastatic pattern as compared to patients with IDC $(3,4)$.

Over the past few years, significant efforts have been done to characterize primary lobular tumors at the genomic, gene expression and immune level(5-9). Genomic studies have provided a more precise estimation of the genomic alterations present in $\mathrm{CDH} 1$, the gene coding for $\mathrm{E}$ cadherin(5-8): approximately 90\% of ILC samples have heterozygous CDH1 deletion, mostly characterized by the loss of the long arm of chromosome 16 , and $\sim 60 \%$ exhibit a mutation in the other allele. Researchers have recently used this characteristic as a potential therapeutic opportunity(10). Indeed, inhibiting ROS1 is synthetically lethal in cells with $C D H 1$ defects, and this strategy is currently being explored in a Phase 2 trial (ClinicalTrials.gov Identifier: NCT03620643). Additionally, the various genomic studies have further highlighted the increased prevalence of mutations in ERpositive/HER2-negative primary ILC in several genes such as AKT1, ARID1A, CDH1, FOXA1, HER2, HER3, PIK3CA, and TBX3 genes as compared to ER-positive/HER2-negative primary IDC(5-8). The gene expression studies have also identified different gene expression subtypes within primary lobular tumors, some with prognostic relevance $(6,7,11)$. And finally, ER-positive lobular tumors have been shown to have significantly lower levels of stromal tumor infiltrating lymphocytes (sTIL) as compared to their ductal counterparts $(9,12)$.

Several large initiatives have recently genomically characterized metastases from patients with breast cancer. These include clinical trials, institutional programs and patient-driven initiatives(1316). While these were not focused on ILC, they have generated and released molecular data on a significant number of these tumors. So far, to the best of our knowledge, only two publications 
specifically reported on ILC metastases(17,18). A recent study used data generated by Foundation Medicine on unmatched primary or metastatic samples from patients with metastatic ILC and reported a critical role of $N F 1$ in mediating endocrine resistance(17). Our group investigated ESRI mutations in metastatic samples from patients with ER-positive ILC and reported a similar frequency and distribution as compared to metastatic samples from patients with ER-positive IDC(18).

While immunotherapy based on checkpoint inhibition is currently being tested in metastatic ILC patients (ClinicalTrials.gov Identifier: NCT03147040), the levels of immune infiltration in ILC metastases remain undetermined. Here, we therefore first centrally characterized the levels of sTIL in a unique European multi-centric series of matched primary and metastatic samples from patients with metastatic ER-positive ILC, further referred to as EuroILC. Secondly, we aimed at characterizing the samples from EuroILC at the genomic level to unravel endocrine resistance and disease progression. We further considered the publicly available genomic data from patients with metastatic ER-positive IDC and ILC from the MSK-IMPACT initiative(13) to identify differences in genomic landscapes of IDC and ILC metastases.

\section{Material and methods}

\section{EuroILC patients and samples}

The patients and samples of this cohort have already been described previously(18). In brief, we considered patients with metastatic ILC from 6 different European hospitals (Institut Jules BordetBrussels, Cliniques Universitaires Saint-Luc-Brussels, GZA Ziekenhuizen-Antwerp, Institut PaoliCalmettes-Marseille, Institut Curie-Paris, Istituto Europeo di Oncologia-Milan) for which primary and metastatic samples were available. Additionally, samples had to fulfil the following criteria: (1) no distinct invasive neoplastic components other than ILC at central revision (mixed ductal-lobular tumors were excluded); (2) ER-positive status of the primary tumor; (3) minimal tumor cellularity of $20 \%$ (if < $20 \%$, then only considered if macrodissection could be done); and, for the DNA analyses (4) availability of >100ng of DNA from a formalin-fixed paraffin-embedded (FFPE) block of the primary 
tumor, metastasis and non-invaded tissue. Priorities in terms of DNA characterization for this project were: first, ddPCR for detecting ESRI mutations(18), second, targeted sequencing, and, third, low pass whole genome sequencing to detect copy-number changes and genomic instability. The project has been approved by the ethics committee of the Institut Jules Bordet $\left(\mathrm{N}^{\circ} 2504\right)$. Given the retrospective nature of the study, the ethics committee did not require the patients to sign an informed consent. The study was performed in accordance with the Declaration of Helsinki.

\section{Histopathological characterization}

Central pathology review was assessed by D.L. for histological type and grade. ER, PgR, HER2 and Ki67 scores were retrieved from the pathology reports. sTIL were assessed by two experienced BC pathologists (R.S and G V.D.E.) using the international guidelines(19). The average of the 2 scores was considered as the final value for that sample. sTIL values were log transformed and when several primary or metastatic samples were available from the same lesion, the median value was considered.

\section{Targeted sequencing}

A TruSeq Custom Amplicon panel of 20 genes frequently mutated in ILC and/or ER-positive $\mathrm{BC}$ in general was designed using DesignStudio from Illumina: AKT1, ARID1A, CDH1, ERBB2, ERBB3, ESR1, FOXA1, GATA3, IGF1R, JAK2, MAP2K4, MAP3K1, NF1, PIK3CA, PTEN, RB1, RUNX1, STAT3, TBX3, and, TP53. A total of 195 tumor samples from 73 patients were sequenced using the Amplicon DS technology from Illumina together with a matched normal sample. Both DNA strands were processed independently, considering the intersection of the called variants between the two strands to limit the noise generated by the FFPE artifacts. Reads were aligned on hg19 using bwa(20). Samples achieving a median coverage of $150 \mathrm{X}$ were kept for downstream analyzes. Normal samples were merged per patient when possible to increase coverage, all normal samples were merged in a pool-of-normal sample to serve as a germline reference for patients without good quality matched normal sample available (15/73=20\% patients). Manta(21) followed by Strelka2(22) were used for the 
calling of both single base substitutions and small somatic insertions-deletions. Possible driver mutations were identified according to Desmedt et al. 2016(5).

\section{Low pass whole genome sequencing and analysis of copy number data}

A total of 165 tumor samples from 63 patients were sequenced with Illumina to an average target coverage of 0.5X. Samples were co-segmented per patient using Copynumber(23), and copy number gains and losses were assessed using ABSOLUTE(24). Solutions were manually selected. In case of inconsistency between the matched primary and metastatic samples, the most reliable sample according to its purity was used to fit the other samples. Samples having a purity of at least $30 \%$ were kept for downstream analyses (Supplementary methods). For the comparisons, we focused on the genes that were reported to be potential 'drivers' by Nik-Zainal et al.(25), as well as CYP19A amplification(26). All the aberrations private to the either one of the matched primary or metastatic samples were manually reviewed. Chromosomal instability (CIN) scores were computed as the ratio between the number of base pair either amplified or deleted over the total number of base pairs analyzed in the cohort.

\section{Publicly available data}

MSK-IMPACT data(13), including clinical data, mutations and raw segmentation data were retrieved from cBioPortal in November 2018 (27). Only female ER-positive patients having experienced a relapse event were selected. ILC and IDC patients were identified according to their oncotree codes (ILC and IDC, BRCANOS, BRCNOS, CSNOS respectively). Possible driver mutations and copy number profiles were determined using the same protocols as for EuroILC.

\section{Statistical analysis}

Association with clinicopathological variables, mutations and copy number alteration frequencies were assessed using Fisher exact tests. Association between continuous sTIL and categorical variables were assessed with Wilcoxon and Kruskal-Wallis tests when having two or more than 2 categories, respectively. When appropriate, a paired Wilcoxon test was performed to consider the matched status of the primary and metastatic samples. Agreement between the two pathologists in 
scoring the sTIL was assessed using the concordance correlation coefficient (CCC) and its confidence interval using the DescTools $\mathrm{R}$ package. Association between genomic alterations and clinicopathological variables were assessed using logistic regression. Median follow-up was computed using the reverse Kaplan-Meier estimator. All tests were considered significant for p-value $<.05$. When appropriate, multiple testing correction were applied using FDR and q-value $<.05$ were considered significant. All analyses were performed under R 3.5.2.

\section{Availability of data and materials}

The BAM files generated in this study are available on Genome-Phenome Archive (EGA) platform (https://ega-archive.org) under accession number EGAS00001004641. In addition, processed data including clinical, mutation and copy number aberration (CNA) data used in this analysis are available in Table S1, Table S3 and Table S4 respectively. More detailed methods can be found in the Data Supplement.

\section{Results}

\section{Patient and sample characteristics}

For the EuroILC cohort, we retrospectively selected 125 patients with metastatic ILC for which both primary and metastatic samples were available from six European institutions. After central pathology review, 94 patients were eligible for matched primary/metastasis sTIL assessment. Sufficient DNA was available for 80 patients to also assess ESRl mutations using droplet digital PCR(18). Additionally, in 73 and 63 patients, respectively, there was sufficient DNA to perform targeted and low pass whole genome sequencing (Figure S1). The clinical and pathological characteristics of the EuroILC cohort $(\mathrm{n}=94)$ are presented in Table 1 and Table S1. In summary, considering the patients eligible for sTIL assessment, $56 \%$ of the patients were menopaused at primary diagnosis, $76 \%$ had a tumor $>2 \mathrm{~cm}, 69 \%$ had axillary lymph node involvement, $44 \%$ of the tumors were of the non-classical histological subtype, $19 \%$ were high grade, $85 \%$ expressed the progesterone receptor (PgR) and $8 \%$ were HER2-amplified. The grading distribution according to the histological 
subtypes was expected and as follows: $31 \%, 63 \%$ and $6 \%$ of the patients having classic ILC of grade 1, 2, 3 respectively; and 3\%,62\% and 35\% of the patients having non-classical ILC of grade 1, 2, 3 respectively. $84 \%$ and $46 \%$ of the patients received adjuvant endocrine therapy and adjuvant chemotherapy, respectively. Loss of hormonal receptor status was observed in the metastatic samples in $11 \%$ and $38 \%$ of the patients for ER and $\mathrm{PgR}$, respectively. The vast majority (84\%) of the metastatic biopsies were taken within the year after metastasis diagnosis.

\section{Stromal tumor-infiltrating lymphocytes in ILC metastases}

In order to estimate the immune infiltration in the primary and metastatic samples from the EuroILC cohort, two experienced pathologists independently scored sTIL using acknowledged guidelines(19). A good concordance was observed (CCC:.89, 95\%CI:.86-.91), leading us to consider the average of the two scores as the final value. We first compared the distribution of sTIL in the primary tumors from EuroILC with the primary tumors from the consecutive multicentric series that were previously analyzed by the same pathologists(9). We observed statistically lower levels in the primary tumor samples from EuroILC (unpaired Wilcoxon test, $\mathrm{p}<.001$, Figure 1A, medians [IQR] of 5\%[3-7] and 1\%[1-7] for Desmedt et al. (9) and EuroILC's primaries, respectively). A paired analysis revealed no significant difference in STIL levels between the paired primary and metastatic samples from EuroILC (paired Wilcoxon test, p=.11, Figure 1A, median [IQR] of 1\% [1-5] for EuroILC's metastases). Higher sTIL in the primary tumor samples from EuroILC were associated with younger age at diagnosis, and with mixed non-classic (which include the so-called pleomorphic tumors, and should not be confused with the mixed ductal-lobular tumors) and trabecular histology (Figure S2A). Higher sTIL in the metastatic samples showed a trend towards association with the mixed non-classic histology (Figure S2B). sTIL infiltration in the ILC metastases did not differ according to the metastatic site (Figure 1B). Immune infiltration is illustrated in Figure $1 \mathrm{C}$ and D for a metastasis in the liver and serosal membrane, respectively.

Clinico- pathological comparison of patient and sample characteristics from EuroILC and MSKIMPAKT 
We compared the clinical and pathological characteristics between patients with metastatic ER-positive ILC from EuroILC and MSK-IMPACT (Table 1, Figure S3, Table S2). The series were comparable with some exceptions: firstly, more than half of the MSK-IMPACT patients (53\%) have high grade tumors as compared to only $19 \%$ of the EuroILC patients, and, secondly, consistent with the retrospective and prospective setting of the two cohorts, more than half of the patients from EuroILC (54\%) already died as compared to $28 \%$ in MSK-IMPACT. This is consistent with the difference in median follow up observed for overall survival between the cohorts: 12.2 years for EuroILC, 5.1 and 8.1 years for the patients with IDC and ILC metastases from MSK-IMPACT, respectively. We also compared the sites of metastatic samples from EuroILC with the metastatic samples from patients with ILC from MSK-IMPACT (Figure S4). The distribution was very similar with the exception of samples from serosal membranes metastases that were more represented in EuroILC and biopsies from chest wall metastases that were more prevalent in MSK-IMPACT. We next compared the sites of metastatic samples between patients with ILC (EuroILC and MSKIMPACT) and IDC (MSK-IMPACT), focusing only on patients with ER-positive tumors, and consistently observed for ILC more samples from the bone, reproductive organs and gastro-intestinal tracts, and less from the liver, lung, distant lymph nodes and soft tissues (Figure S4).

\section{Genomic comparison of matched primary and metastatic EuroILC samples}

The individual clinical and pathological characteristics of the 43 EuroILC patients that have genomic data and passed quality control for both their primary and metastatic samples are represented in Figure 2. For $>50 \%$ of patients, either the primary or the metastatic sample(s) did not pass QC because the DNA was too degraded (Figure S1). We first compared the mutational landscape between matched primary and metastatic samples (32 patients). Of note, when multiple primary samples per patient were sequenced, which was the case in $16 / 32(50 \%)$ of patients, then the mutation was considered to be present if it was detected in at least one of the samples. A similar rule was applied for the metastatic samples, concerning also $16 / 32(50 \%)$ patients, with multiple metastatic samples being mostly available from the same metastatic lesion. As displayed in the heatmap in Figure 3A, genes mutated in $>10 \%$ of either primary and/or metastatic samples were in decreasing order: $C D H 1$, 
PIK3CA, TBX3, MAP3K1, TP53, GATA3, ESR1, ERBB2, ARIDIA, IGF1R, FOXA1, and AKT1. Lolipop plots representing the distribution of mutations in some of these genes are illustrated in Figure 3B. We observed mutations private to the metastatic sample(s), i.e. mutations only detected in the metastatic but not in the matched primary sample(s), in $\mathrm{CDH} 1$ in $7 / 32$ (with 2/7 that also have another shared $C D H 1$ mutation in their primary and metastatic samples), in ARIDIA, ERBB2 and ESR1 in 2/32 (6\%) of the patients, and in AKT1, GATA3, MAPK3K1, NF1, RB1 and RUNX1 in 1/32 (3\%) of the patients. Mutations private to the metastasis were also seen in two patients where the metastatic sample lost ER expression (patient 32 and 36). Of note, loss of several mutations that were present in the primary tumor was observed in the metastatic samples from some patients. This could be explained by tumor heterogeneity, since some of these mutations were only present at a low allele frequency (such as ERBB3 D501N mutation in patient 9, seen at a VAF of $8 \%$ only in 1 of the 2 primary samples, not in the metastatic sample) or in only some of the primary tumor samples (ERBB2 L755S mutation in patient 1 , seen at a VAF of $51 \%$ in only one of the 5 primary samples, not in the metastatic sample) (Figure S5). With regard to copy number alterations (Figure 3 C-D), results were available for 21 matched primary-metastasis patients. Here again, as for the mutations, we observed copy number alterations private to the metastatic sample(s), such as MAP2K4 and NCORI deletions in 4/21 (19\%) patients, TP53 deletion in 3/21 (14\%) patients, PTEN and AKT1 deletions in 2/21 (10\%) patients, as well as $C C N D 1$ and $C C N E 1$ amplifications in 2/21 (10\%) patients (Figure S6). Figure 4 represents the disease evolution and the treatments received of three patients, together with the private alterations observed in each sample.

We further computed chromosomal instability for all primary and metastatic samples, and computed for each patient the change in instability (Figure 3E-G). We observed a positive but not statistically significant association between the primary/metastatic time difference in sampling and the primary/metastatic difference in chromosomal instability (Spearman $r h o=0.34, p=.14$ ), suggesting that the longer the disease evolution lasts, the more the metastasis differs from the primary tumor.

Comparison of the genomic landscape of ILC and IDC metastases from EuroILC and MSKIMPACT 
We started by comparing the genomic alterations present in the primary and metastatic ILC samples from EuroILC (from 54 and 40 patients, respectively) and MSK-IMPACT (from 51 and 135 patients, respectively), to see whether these were comparable despite the clinical and pathological differences reported above (Figure 5A-B, Figure S7). Overall, ILC samples from both cohorts were comparable. Nevertheless, we observed an increased frequency in copy number losses in $A K T 1$, $B R C A 2$ and $R B 1$ in the primary samples from EuroILC as compared to the primary ILC samples from MSK-IMPACT, and an increased frequency in IGFIR mutations and copy number losses in $A K T 1$ and MAP3K1 in the metastases from EuroILC as compared to those from MSK-IMPACT. To identify genes that are differentially altered between ER-positive ILC and IDC metastases, we first compared the mutations and CNAs present in the samples from the EuroILC patients with the ones from the MSK-IMPACT patients with IDC (260 with primary samples and 563 with metastatic samples, Figure 5C-D, Figure S7). We then repeated the comparison between the ILC and IDC metastases from MSKIMPACT (Figure 5E-F, Figure S7). In terms of mutations, the two comparisons consistently identified a significant increased prevalence of mutations affecting $C D H 1, E R B B 2, F O X A 1$ and TBX3, and a decreased prevalence in TP53 mutations in ILC metastases as compared to IDC metastases. Additionally, GATA3 mutations were further more prevalent in IDC metastases, although it only reached statistical significance in the MSK-IMPACT comparison. Finally, IGFIR mutations were significantly more prevalent in ILC metastases but only when considering the EuroILC cohort. No difference in prevalence was however observed with regard to PIK3CA or ESRI mutations. With regard to copy number aberrations, $C D H 1$ deletions were, as expected, consistently more frequent, in the primary and metastatic samples from ILC as compared to IDC. Additional copy number differences were further observed but only in the comparison involving either the EuroILC or MSKIMPACT ILC cohort (Figure S7). Of note, no difference was observed with regard to the prevalence of TP53 deletions.

\section{Discussion}


In this study, we assembled retrospectively matched primary and metastatic samples from patients with metastatic ILC to evaluate the levels of immune infiltrates in the metastatic disease and to investigate de novo and acquired genomic alterations associated with endocrine resistance. $\mathrm{We}$ further aimed at comparing the genomic landscape of metastases from patients with ERpositive/HER2-negative ILC and ER-positive/HER2-negative IDC, using the publicly available MSKIMPACT series(13).

In EuroILC, we observed a loss of ER and PgR in the metastases from $11 \%$ and $38 \%$ of the patients, respectively. This is in line with what has been reported so far in the literature for endocrineresistant breast cancer(28). With regard to the site of metastatic sampling in patients with ILC, our results are consistent with Sokol et al.(17) and in agreement with previous reports that demonstrated that after accounting for hormone receptor status, patients with ILC had less lung and liver metastases but more ovarian and gastrointestinal metastases both at first site and overall, as compared to patients with $\operatorname{IDC}(3,4)$.

We previously characterized immune infiltrates in primary tumors from patients with ILC and reported lower levels of sTIL in ER-positive/HER2-negative ILC as compared to ER-positive/HER2negative IDC $(9,12)$. Here, we observed even lower levels of sTIL in the primary tumors from the EuroILC cohort as compared to our previous consecutive series of primary ILC. There was no difference in sTIL levels between the primary and matched metastasi(e)s, all being globally very low. We nevertheless observed higher sTIL levels both in the primary tumors and the metastases of the mixed non-classic histological subtype, which encompasses the so-called pleomorphic tumors. There was no apparent distinction between the various metastatic sites, although numbers were too small to apply statistical tests. A more detailed characterization of these immune cells is needed to better identify patients who may benefit from immunotherapy.

Several somatic genomic alterations associated with endocrine resistance have been uncovered and have recently been summarized by Hanker et al.(29). In EuroILC, 21/40 (53\%) of the patients were harboring at least one mutation in their metastases which was previously reported to be 
associated with endocrine resistance, such as $A K T 1(30,31), \operatorname{ARIDIA}(13,32), \operatorname{ERBB2}(13,30,33)$, ESRI(34,35), FOXAl(13), NFl(13,17), or PTEN(36) mutations. A similar frequency was observed in the MSK-IMPACT ILC cohort, namely 56/135 (41\%). Of interest, 7/32 (22\%) of the patients from the EuroILC cohort had private point mutations in the metastatic sample(s) in the $\operatorname{AKTI}(30,31)$, $\operatorname{ARIDIA}(13,32), \operatorname{ERBB2}(13,30,33), \operatorname{ESR} 1(34,35)$ or $N F 1(13,17,37)$ gene. If we consider CNAs, then 12/21 had at least one event associated with endocrine resistance, such as ERBB2, CYP19A1(26), EGFR(13), FGFRI(38) or MYC(13) amplification, or PTEN(36) or NF1 deletion. We further observed aberrations private to the metastasis in $4 / 21(19 \%)$ patients, such as $\operatorname{PTEN}(36)$ or $\operatorname{NF} 1(13,17,37)$ deletion or CYP19A1(26) amplification. While the fact of observing mutations only in metastases and not in the matched primary samples is suggestive of acquired resistance, this cannot be formally proved as minor subclones might have been present in a part of the primary tumor that has not been sequenced. Altogether, as several of these alterations are targetable(29) it implies that a characterization of the metastatic disease, either by sequencing a metastatic biopsy or circulating tumor DNA (ctDNA) as in the PlasmaMATCH trial(31), is necessary to personalize the treatment of patients with metastatic ILC.

Some observations could be made when comparing the genomic landscape of metastases from patients with ER-positive ILC and IDC such as an enrichment of mutations affecting $C D H 1, E R B B 2$, FOXA1 and TBX3 genes, an increase in $C D H 1$ deletions, and a decreased prevalence in TP53 mutations, but not TP53 deletions, in ILC metastases as compared to IDC metastases. Of interest we observed an increased frequency of IGFRI mutations in metastases from the EuroILC cohort. While these would still need to be functionally characterized, Nagle et al. demonstrated that the hyperactivation of the IGF1R pathway was more frequent in ILC as compared to IDC primary tumors and that this could result in increased sensitivity to IGF1R/InsR targeted therapy(39). We did not detect an increased prevalence of NF1 mutations or deletions in metastases from patients with ILC, as suggested by Sokol et al.(17). Further, it is important to report that we did not observe a difference in the frequency of PIK3CA mutations between ILC and IDC metastases, a finding that is clinically relevant since the first PI3K $\alpha$-specific inhibitor alpelisib is now available to treat patients with hormone receptor-positive/HER2-negative advanced breast cancer (40). In this context, it is however 
important to remember that previous studies have demonstrated that the PI3K/Akt pathway can be activated in ILC independently of oncogenic mutations in this pathway $(6,41)$.

Our study has several limitations. Firstly, the immune characterization has here so far been limited to the standard evaluation of sTIL(19). A more detailed and spatial evaluation of each cell type from the tumor microenvironment is however needed(29). Secondly, for most of the patients only one metastatic lesion was biopsied. Acknowledging the intra-patient inter-metastases genomic heterogeneity which has been highlighted in several autopsy-based studies(13,42-48), we can reasonably assume to have missed some genomic alterations present in other metastatic sites or in other regions of the biopsied metastasis. Importantly, this intra-patient inter-metastases heterogeneity has also been shown to affect genes involved in treatment resistance such as ESRI and PTEN mutations $(13,42,47)$. Thirdly, the rather limited panel size for mutational analysis may have prevented us from discovering and/or confirming recent ILC specific driver genes that are present at a low frequency in the tumors of patients with ILC, such as mutations in FGFR4(49) or BRAF, CTCF, $K R A S$, and $M A P 2 K 1(13)$. Fourthly, our study did not consider additional non-genomic elements that could be associated with endocrine resistance in ILC, such as lipid metabolism which has previously been suggested as a potential mechanism by cell line experiments(50). Our results suggest however that somatic alterations could already explain endocrine resistance in more than half of the patients with metastatic ILC. A final limitation relates to the number of patients in EuroILC. This exemplifies the difficulty in assembling retrospectively a cohort of patients with ILC with sufficient and good quality DNA from matched primary and metastatic samples. This represents however a general need and challenge for the general breast cancer community and not only for patients with ILC(29).

To conclude, firstly, while most of the ILC metastases present low or no immune infiltration, higher levels can be observed in tumors from the mixed non-classic histology. Whether or not these patients represent potential candidates for immunotherapy requires additional investigation. Secondly, we do have evidence of possibly acquired alterations associated with endocrine resistance during disease evolution in approximately one out of five patients with ILC. While the present results still need to be confirmed in larger cohorts of metastatic ILC patients, they support the need to characterize the metastatic disease, either using a metastatic biopsy or ctDNA, to refine treatment for these patients. 
Additionally, the identified genomic alterations may not only be associated with endocrine resistance, but could also predict resistance to additional treatments. For instance, PTEN alterations have recently been associated with resistance to PI3K $\alpha$ and CDK4/6 inhibitors(51) and FGFR1 amplifications have been shown to mediate resistance to CDK4/6 inhibitors(52). Thirdly, while we acknowledge the presence of non-genomic mechanisms(29), genomic alterations are potentially explaining endocrine resistance in a large proportion of ILC patients. Finally, the genomic landscape of ILC metastases does present some differences with regard to IDC metastases. Some of those, such as ERBB2 and FOXAI mutations, have been related to endocrine resistance. 


\section{List of abbreviations}

CCC: concordance correlation coefficient, CIN score: chromosomal instability score, CNA: copy number aberration, ctDNA: circulating tumor DNA, ER: estrogen receptor, FFPE : formalin-fixed paraffin-embedded, IDC: invasive ductal breast cancer, ILC: invasive lobular breast cancer, PgR: progesterone receptor, sTIL: stromal tumour infiltrating lymphocytes.

\section{Acknowledgements}

This work has been supported by "Les Amis de Bordet", the "Fondation contre le cancer" (FAFC/2016/762), "The Breast Cancer Research Foundation", and the Belgian Fonds National de la Recherche Scientifique (F.R.S-FNRS). F. Richard. is supported by a grant from the "Fondation Cancer Luxembourg" (FC/2018/07). C. Marchio was supported in part by a grant from the Mayent-Rothschild Foundation to Institut Curie.

The authors thank the patients and their families, the Biobanks from all participating hospitals, David Brown, Sophia Leduc, Imane Bachir, Yacine Bareche, Marion Maetens, Floriane Dupont, Dominique Roels and Jeanne Letor for their technical and clinical support. 


\section{References}

1. McCart Reed AE, Kutasovic JR, Lakhani SR, Simpson PT. Invasive lobular carcinoma of the breast: morphology, biomarkers and 'omics. Breast Cancer Res. 2015;17:12.

2. Pestalozzi BC, Zahrieh D, Mallon E, Gusterson BA, Price KN, Gelber RD, et al. Distinct clinical and prognostic features of infiltrating lobular carcinoma of the breast: combined results of 15 International Breast Cancer Study Group clinical trials. J Clin Oncol. 2008;26:3006-14.

3. Mathew A, Rajagopal PS, Villgran V, Sandhu GS, Jankowitz RC, Jacob M, et al. Distinct Pattern of Metastases in Patients with Invasive Lobular Carcinoma of the Breast. Geburtshilfe Frauenheilkd. 2017;77:660-6.

4. Arpino G, Bardou VJ, Clark GM, Elledge RM. Infiltrating lobular carcinoma of the breast: tumor characteristics and clinical outcome. Breast Cancer Res. 2004;6:R149.

5. Desmedt C, Zoppoli G, Gundem G, Pruneri G, Larsimont D, Fornili M, et al. Genomic Characterization of Primary Invasive Lobular Breast Cancer. J Clin Oncol. 2016; 34:1872-81.

6. Ciriello G, Gatza ML, Beck AH, Wilkerson MD, Rhie SK, Pastore A, et al. Comprehensive Molecular Portraits of Invasive Lobular Breast Cancer. Cell. 2015;163:506-19.

7. Michaut M, Chin SF, Majewski I, Severson TM, Bismeijer T, de Koning L, et al. Integration of genomic, transcriptomic and proteomic data identifies two biologically distinct subtypes of invasive lobular breast cancer. Sci Rep. 2016;6:18517.

8. Pereira B, Chin SF, Rueda OM, Vollan HK, Provenzano E, Bardwell HA, et al. The somatic mutation profiles of 2,433 breast cancers refines their genomic and transcriptomic landscapes. Nat Commun. 2016;7:11479.

9. Desmedt C, Salgado R, Fornili M, Pruneri G, Van den Eynden G, Zoppoli G, et al. Immune Infiltration in Invasive Lobular Breast Cancer. JNCI J Natl Cancer Inst. 2018; 110:768-76.

10. Bajrami I, Marlow R, van de Ven M, Brough R, Pemberton HN, Frankum J, et al. ECadherin/ROS1 Inhibitor Synthetic Lethality in Breast Cancer. Cancer Discov. American 
Association for Cancer Research; 2018;8:498-515.

11. Du T, Zhu L, Levine KM, Tasdemir N, Lee A V., Vignali DAA, et al. Invasive lobular and ductal breast carcinoma differ in immune response, protein translation efficiency and metabolism. Sci Rep. Nature Publishing Group; 2018;8.

12. Tille JC, Vieira AF, Saint-Martin C, Djerroudi L, Furhmann L, Bidard FC, et al. Tumorinfiltrating lymphocytes are associated with poor prognosis in invasive lobular breast carcinoma. Mod Pathol. Springer Nature; 2020; 1-10.

13. Razavi P, Chang MT, Xu G, Bandlamudi C, Ross DS, Vasan N, et al. The Genomic Landscape of Endocrine-Resistant Advanced Breast Cancers. Cancer Cell. 2018;34:427-438.e6.

14. Bertucci F, Ng CKY, Patsouris A, Droin N, Piscuoglio S, Carbuccia N, et al. Genomic characterization of metastatic breast cancers. Nature. 2019;569:560-4.

15. Wagle N, Painter C, Anastasio E, Dunphy M, McGillicuddy M, Kim D, et al. The Metastatic Breast Cancer (MBC) project: Accelerating translational research through direct patient engagement. J Clin Oncol. 2018;35:1076-1076.

16. Angus L, Smid M, Wilting SM, van Riet J, Van Hoeck A, Nguyen L, et al. The genomic landscape of metastatic breast cancer highlights changes in mutation and signature frequencies. Nat Genet. Nature Publishing Group; 2019;51:1450-8.

17. Sokol ES, Feng YX, Jin DX, Basudan A, Lee A V, Atkinson JM, et al. Loss of function of NF1 is a mechanism of acquired resistance to endocrine therapy in lobular breast cancer. Ann Oncol. 2018; 30:115-23.

18. Desmedt C, Pingitore J, Rothé F, Marchio C, Clatot F, Rouas G, et al. ESR1 mutations in metastatic lobular breast cancer patients. npj Breast Cancer. Springer Nature; 2019;5.

19. Salgado R, Denkert C, Demaria S, Sirtaine N, Klauschen F, Pruneri G, et al. The evaluation of tumor-infiltrating lymphocytes (TILs) in breast cancer: recommendations by an International TILs Working Group 2014. Ann Oncol. 2014;26:259-71. 
20. Li H, Durbin R. Fast and accurate long-read alignment with Burrows-Wheeler transform. Bioinformatics. 2010;26:589-95.

21. Chen X, Schulz-Trieglaff O, Shaw R, Barnes B, Schlesinger F, Källberg M, et al. Manta: Rapid detection of structural variants and indels for germline and cancer sequencing applications. Bioinformatics. 2016;32:1220-2.

22. Kim S, Scheffler K, Halpern AL, Bekritsky MA, Noh E, Källberg M, et al. Strelka2: Fast and accurate variant calling for clinical sequencing applications. bioRxiv. 2017;192872.

23. Nilsen G, Liestøl K, Loo P Van, Moen Vollan HK, Eide MB, Rueda OM, et al. Copynumber: Efficient algorithms for single- and multi-track copy number segmentation. BMC Genomics. $2012 ; 13: 1-16$.

24. Carter SL, Cibulskis K, Helman E, McKenna A, Shen H, Zack T, et al. Absolute quantification of somatic DNA alterations in human cancer. Nat Biotechnol. 2012; 30:413-21.

25. Nik-Zainal S, Davies H, Staaf J, Ramakrishna M, Glodzik D, Zou X, et al. Landscape of somatic mutations in 560 breast cancer whole-genome sequences. Nature. 2016;534:47-54.

26. Magnani L, Frigè G, Gadaleta RM, Corleone G, Fabris S, Kempe H, et al. Acquired CYP19A1 amplification is an early specific mechanism of aromatase inhibitor resistance in ER $\alpha$ metastatic breast cancer. Nat Genet. Nature Publishing Group; 2017;49:444-50.

27. Cerami E, Gao J, Dogrusoz U, Gross BE, Sumer SO, Aksoy BA, et al. The cBio Cancer Genomics Portal: An open platform for exploring multidimensional cancer genomics data. Cancer Discov. 2012;2:401-4.

28. Shiino S, Kinoshita T, Yoshida M, Jimbo K, Asaga S, Takayama S, et al. Prognostic Impact of Discordance in Hormone Receptor Status Between Primary and Recurrent Sites in Patients With Recurrent Breast Cancer. Clin Breast Cancer. Elsevier Inc.; 2016;16:e133-40.

29. Hanker AB, Sudhan DR, Arteaga CL. Overcoming Endocrine Resistance in Breast Cancer. Cancer Cell. Elsevier; 2020;37:496-513. 
30. Desmedt C, Zoppoli G, Gundem G, Pruneri G, Larsimont D, Fornili M, et al. Genomic Characterization of Primary Invasive Lobular Breast Cancer. J Clin Oncol. 2016;34:1872-80.

31. Turner N, Kingston B, Kilburn L, Kernaghan S, Wardley AM, Macpherson I, et al. Abstract GS3-06: Results from the plasmaMATCH trial: A multiple parallel cohort, multi-centre clinical trial of circulating tumour DNA testing to direct targeted therapies in patients with advanced breast cancer (CRUK/15/010). Cancer Res. American Association for Cancer Research (AACR); 2020. page GS3-06-GS3-06.

32. Xu G, Chhangawala S, Cocco E, Razavi P, Cai Y, Otto JE, et al. ARID1A determines luminal identity and therapeutic response in estrogen-receptor-positive breast cancer. Nat Genet. Nature Research; 2020;52:198-207.

33. Nayar U, Cohen O, Kapstad C, Cuoco MS, Waks AG, Wander SA, et al. Acquired HER2 mutations in ER+ metastatic breast cancer confer resistance to estrogen receptor-directed therapies. Nat Genet. 2019;51:207-16.

34. Jeselsohn R, Yelensky R, Buchwalter G, Frampton G, Meric-Bernstam F, Gonzalez-Angulo $\mathrm{AM}$, et al. Emergence of constitutively active estrogen receptor-alpha mutations in pretreated advanced estrogen receptor-positive breast cancer. Clin Cancer Res. 2014;20:1757-67.

35. Jeselsohn R, De Angelis C, Brown M, Schiff R. The Evolving Role of the Estrogen Receptor Mutations in Endocrine Therapy-Resistant Breast Cancer. Curr Oncol Rep. 2017;19:35.

36. Fu X, Creighton CJ, Biswal NC, Kumar V, Shea M, Herrera S, et al. Overcoming endocrine resistance due to reduced PTEN levels in estrogen receptor-positive breast cancer by cotargeting mammalian target of rapamycin, protein kinase $\mathrm{B}$, or mitogen-activated protein kinase kinase. Breast Cancer Res. BioMed Central Ltd.; 2014;16:430.

37. Pearson A, Proszek P, Pascual J, Fribbens C, Shamsher MK, Kingston B, et al. Inactivating NF1 mutations are enriched in advanced breast cancer and contribute to endocrine therapy resistance. Clin Cancer Res. American Association for Cancer Research Inc.; 2020;26:608-22. 
38. Giltnane JM, Hutchinson KE, Stricker TP, Formisano L, Young CD, Estrada M V., et al. Genomic profiling of ER+ breast cancers after short-term estrogen suppression reveals alterations associated with endocrine resistance. Sci Transl Med. American Association for the Advancement of Science; 2017;9.

39. Nagle AM, Levine KM, Tasdemir N, Scott JA, Burlbaugh K, Kehm J, et al. Loss of E-cadherin enhances IGF1-IGF1R pathway activation and sensitizes breast cancers to anti-IGF1R/InsR inhibitors. Clin Cancer Res. American Association for Cancer Research Inc.; 2018;24:5165-77.

40. André F, Ciruelos E, Rubovszky G, Campone M, Loibl S, Rugo HS, et al. Alpelisib for PIK3CA-mutated, hormone receptor-positive advanced breast cancer. N Engl J Med. Massachussetts Medical Society; 2019;380:1929-40.

41. Teo K, Gómez-Cuadrado L, Tenhagen M, Byron A, Rätze M, van Amersfoort M, et al. Ecadherin loss induces targetable autocrine activation of growth factor signalling in lobular breast cancer. Sci Rep. Nature Publishing Group; 2018;8.

42. Juric D, Castel P, Griffith M, Griffith OL, Won HH, Ellis H, et al. Convergent loss of PTEN leads to clinical resistance to a PI(3)Kalpha inhibitor. Nature. 2014;518:240-4.

43. Hoadley KA, Siegel MB, Kanchi KL, Miller CA, Ding L, Zhao W, et al. Tumor Evolution in Two Patients with Basal-like Breast Cancer: A Retrospective Genomics Study of Multiple Metastases. PLoS Med. 2016;13:e1002174.

44. Savas P, Teo ZL, Lefevre C, Flensburg C, Caramia F, Alsop K, et al. The Subclonal Architecture of Metastatic Breast Cancer: Results from a Prospective Community-Based Rapid Autopsy Program “CASCADE.” Ladanyi M, editor. PLOS Med. 2016;13:e1002204.

45. Brown D, Smeets D, Székely B, Larsimont D, Szász AM, Adnet P-Y, et al. Phylogenetic analysis of metastatic progression in breast cancer using somatic mutations and copy number aberrations. Nat Commun. 2017;8:14944.

46. Avigdor BE, Cimino-Mathews A, DeMarzo AM, Hicks JL, Shin J, Sukumar S, et al. 
Mutational profiles of breast cancer metastases from a rapid autopsy series reveal multiple evolutionary trajectories. JCI insight. 2017;2.

47. De Mattos-Arruda L, Sammut S-J, Ross EM, Markowetz F, Seoane J, Caldas Correspondence C. The Genomic and Immune Landscapes of Lethal Metastatic Breast Cancer. Cell Rep. 2019;27.

48. Siegel MB, He X, Hoadley KA, Hoyle A, Pearce JB, Garrett AL, et al. Integrated RNA and DNA sequencing reveals early drivers of metastatic breast cancer. J Clin Invest. 2018;128:1371-83.

49. Levine KM, Priedigkeit N, Basudan A, Tasdemir N, Sikora MJ, Sokol ES, et al. FGFR4 overexpression and hotspot mutations in metastatic er+ breast cancer are enriched in the lobular subtype. npj Breast Cancer. Nature Research; 2019;5:1-5.

50. Du T, Sikora MJ, Levine KM, Tasdemir N, Riggins RB, Wendell SG, et al. Key regulators of lipid metabolism drive endocrine resistance in invasive lobular breast cancer. Breast Cancer Res. BioMed Central Ltd.; 2018;20:106.

51. Costa C, Wang Y, Ly A, Hosono Y, Ellen M, Walmsley CS, et al. PTEN loss mediates clinical cross-resistance to CDK4/6 and PI3K $\alpha$ inhibitors in breast cancer. Cancer Discov. American Association for Cancer Research (AACR); 2019;CD-18-0830.

52. Formisano L, Lu Y, Servetto A, Hanker AB, Jansen VM, Bauer JA, et al. Aberrant FGFR signaling mediates resistance to CDK4/6 inhibitors in ER+ breast cancer. Nat Commun. Nature Publishing Group; 2019;10:1373. 
Table 1: Patient and sample characteristics of the EuroILC, MSK-IMPACT ILC and IDC cohorts. Numbers represent the counts and percentage are shown in brackets. Differences in distributions between cohorts are screened by Fisher tests and p-values are reported in the right columns. Of note, when multiple metastatic samples were available for a patient, we arbitrarily reported the ER, PgR and HER2 status from the latest one. Abbreviations: AI: aromatase inhibitors, ET: endocrine therapy, ER: estrogen receptor, G: rade, PgR: progesterone status, SERM: Specific Estrogen-Receptor Modulators. 


\begin{tabular}{|c|c|c|c|c|c|c|}
\hline & $\begin{array}{l}\text { EuroILC } \\
(94 \mathrm{pts})\end{array}$ & $\begin{array}{l}\text { M MSK- } \\
\text { IMPACT } \\
\text { ILC (135 pts) }\end{array}$ & $\begin{array}{l}\text { M MSK- } \\
\text { IMPACT } \\
\text { IDC (563 pts) }\end{array}$ & $\begin{array}{l}\text { EuroILC } \\
\text { vs MSK } \\
\text { ILC }\end{array}$ & $\begin{array}{l}\text { EuroILC } \\
\text { vs MSK } \\
\text { IDC }\end{array}$ & $\begin{array}{l}\text { MSK } \\
\text { ILC } \\
\text { vs } \\
\text { IDC } \\
\end{array}$ \\
\hline \multicolumn{7}{|c|}{ Age at diagnosis } \\
\hline$<50$ & $28(30 \%)$ & $51(38 \%)$ & $305(54 \%)$ & 0.26 & $<0.001$ & $<0.001$ \\
\hline$\geq \mathbf{5 0}$ & $66(70 \%)$ & $84(62 \%)$ & $258(46 \%)$ & & & \\
\hline \multicolumn{7}{|c|}{ Menopausal status } \\
\hline Pre/peri & $41(44 \%)$ & $67(50 \%)$ & $351(63 \%)$ & 0.43 & $<0.001$ & $<0.01$ \\
\hline Post & $53(56 \%)$ & $67(50 \%)$ & $209(37 \%)$ & & & \\
\hline Missing & 0 & 1 & 3 & & & \\
\hline \multicolumn{7}{|c|}{$\begin{array}{l}\text { Histological primary tumor } \\
\text { size }\end{array}$} \\
\hline$<2 \mathrm{~cm}$ & $21(24 \%)$ & $43(35 \%)$ & $210(44 \%)$ & 0.04 & $<0.001$ & 0.08 \\
\hline$\geq 2 \mathrm{~cm}$ & $67(76 \%)$ & $79(65 \%)$ & $263(56 \%)$ & & & \\
\hline Missing & 6 & 13 & 90 & & & \\
\hline \multicolumn{7}{|l|}{ Nodal status } \\
\hline Negative & $29(31 \%)$ & $40(33 \%)$ & $194(40 \%)$ & 0.88 & 0.13 & 0.18 \\
\hline Positive & $64(69 \%)$ & $82(67 \%)$ & $293(60 \%)$ & & & \\
\hline Missing & 1 & 13 & 76 & & & \\
\hline \multicolumn{7}{|c|}{ Histological subtype } \\
\hline Classic & $51(56 \%)$ & NA & NA & NA & & NA \\
\hline Non-classic & $40(44 \%)$ & NA & NA & & & \\
\hline Missing & 3 & & & & & \\
\hline \multicolumn{7}{|c|}{ Histological grade (primary) } \\
\hline G1/G2 & $75(81 \%)$ & $34(47 \%)$ & $139(27 \%)$ & $<0.001$ & $<0.001$ & $<0.001$ \\
\hline G3 & $18(19 \%)$ & $38(53 \%)$ & $382(73 \%)$ & & & \\
\hline Missing & 1 & 63 & 42 & & & \\
\hline \multicolumn{7}{|c|}{ PgR status (primary) } \\
\hline Negative & $14(15 \%)$ & $23(18 \%)$ & $109(19 \%)$ & 0.72 & 0.47 & 0.71 \\
\hline Positive & $77(85 \%)$ & $108(82 \%)$ & $453(81 \%)$ & & & \\
\hline Missing & 3 & 4 & 1 & & & \\
\hline \multicolumn{7}{|c|}{ HER2 status (primary) } \\
\hline Negative & $78(92 \%)$ & $119(94 \%)$ & $448(86 \%)$ & 0.57 & 0.22 & 0.01 \\
\hline Positive & $7(8 \%)$ & $7(6 \%)$ & $70(14 \%)$ & & & \\
\hline Missing & 9 & 9 & 45 & & & \\
\hline \multicolumn{7}{|c|}{ Adjuvant chemotherapy } \\
\hline No & $51(54 \%)$ & $77(57 \%)$ & $292(52 \%)$ & 0.69 & 0.74 & 0.29 \\
\hline Yes & $43(46 \%)$ & $58(43 \%)$ & $271(48 \%)$ & & & \\
\hline Missing & 0 & 0 & 0 & & & \\
\hline \multicolumn{7}{|c|}{ Adjuvant endocrine therapy } \\
\hline No & $15(16 \%)$ & $31(23 \%)$ & $182(32 \%)$ & 0.24 & 0.001 & 0.04 \\
\hline Yes & $78(84 \%)$ & $104(77 \%)$ & $381(68 \%)$ & & & \\
\hline Missing & 1 & 0 & 0 & & & \\
\hline
\end{tabular}




\begin{tabular}{|c|c|c|c|c|c|c|}
\hline Adjuvant radiotherapy & & & & & & \\
\hline No & $27(30 \%)$ & NA & NA & NA & NA & NA \\
\hline Yes & $64(70 \%)$ & NA & NA & & & \\
\hline Missing & 3 & & & & & \\
\hline \multicolumn{7}{|l|}{ Time to relapse } \\
\hline Median & $4.69 \mathrm{yrs}$ & $4.65 \mathrm{yrs}$ & $3.53 \mathrm{yrs}$ & & & \\
\hline De novo metastatic & $14(15 \%)$ & $24(18 \%)$ & $109(19 \%)$ & 0.58 & 0.17 & 0.24 \\
\hline Relapsed $<5$ years & $34(36 \%)$ & $46(34 \%)$ & $235(42 \%)$ & & & \\
\hline Relapsed $>5$ but $<10$ years & $31(33 \%)$ & $36(27 \%)$ & $126(22 \%)$ & & & \\
\hline Relapsed $>10$ years & $15(16 \%)$ & $29(21 \%)$ & $93(17 \%)$ & & & \\
\hline \multicolumn{7}{|l|}{ ER status (metastasis) } \\
\hline Negative & $9(11 \%)$ & $16(13 \%)$ & $71(13 \%)$ & 0.83 & 0.72 & 1.00 \\
\hline Positive & $73(89 \%)$ & $108(87 \%)$ & $471(87 \%)$ & & & \\
\hline Missing & 12 & 11 & 21 & & & \\
\hline \multicolumn{7}{|l|}{ PgR status (metastasis) } \\
\hline Negative & $38(48 \%)$ & $59(50 \%)$ & $249(47 \%)$ & 0.89 & 0.81 & 0.61 \\
\hline Positive & $41(52 \%)$ & $60(50 \%)$ & $285(53 \%)$ & & & \\
\hline Missing & 15 & 16 & 29 & & & \\
\hline \multicolumn{7}{|l|}{ HER2 status (metastasis) } \\
\hline Negative & $75(95 \%)$ & $109(92 \%)$ & $463(88 \%)$ & 0.41 & 0.08 & 0.42 \\
\hline Positive & $4(5 \%)$ & $10(8 \%)$ & $61(12 \%)$ & & & \\
\hline Missing & 15 & 16 & 39 & & & \\
\hline \multicolumn{7}{|l|}{ Timing metastatic biopsy } \\
\hline$<1$ year diagnosis & $75(84 \%)$ & NA & NA & NA & NA & NA \\
\hline Later & $14(16 \%)$ & NA & NA & & & \\
\hline Missing & 5 & & & & & \\
\hline \multicolumn{7}{|l|}{$\begin{array}{l}\text { ET before metastatic } \\
\text { sampling }\end{array}$} \\
\hline SERM & $39(41 \%)$ & $34(25 \%)$ & $185(33 \%)$ & 0.01 & $<0.001$ & $<0.001$ \\
\hline AI only & $20(21 \%)$ & $39(29 \%)$ & $110(20 \%)$ & & & \\
\hline SERM and AI & $28(30 \%)$ & $35(26 \%)$ & $92(16 \%)$ & & & \\
\hline No ET & $7(7 \%)$ & $27(20 \%)$ & $176(31 \%)$ & & & \\
\hline \multicolumn{7}{|l|}{$\begin{array}{l}\text { Duration ET before } \\
\text { metastatic sampling }\end{array}$} \\
\hline$<2$ years & $17(20 \%)$ & $16(15 \%)$ & $101(26 \%)$ & 0.54 & 0.36 & 0.01 \\
\hline $2-4$ years & $20(23 \%)$ & $22(20 \%)$ & $94(24 \%)$ & & & \\
\hline$>4$ years & $50(57 \%)$ & $70(65 \%)$ & $192(50 \%)$ & & & \\
\hline \multicolumn{7}{|l|}{ Deceased } \\
\hline No & $43(46 \%)$ & $99(73 \%)$ & $424(75 \%)$ & $<0.001$ & $<0.001$ & 0.66 \\
\hline Yes & $51(54 \%)$ & $36(27 \%)$ & $139(25 \%)$ & & & \\
\hline
\end{tabular}




\section{Figures}

Figure 1: sTIL landscape in ILC specimens. (A) Comparison of EuroILC primary sTIL (58 patients) with the Desmedt, et al. 2018 ILC primary sTIL (577 patients) (9) and EuroILC metastasis sTIL (67 patients). Significance of the differences are assessed by a Wilcoxon unpaired test between EuroILC primaries and Desmedt, et al 2018 ILC primaries and a paired test between EuroILC primaries and EuroILC metastases. The median value is considered if several samples are available per patient. (B) sTIL infiltration according to the metastatic site. (C,D) Scanned H\&E slides from two EuroILC metastases: (C) liver sample (4M2) with 70\% sTIL; (D) serosal membrane sample (24M1) with $22.5 \%$ sTIL. Abbreviations: GI: gastrointestinal, mb: membrane.

Figure 2: Overview of the patient and sample characteristics from EuroILC for the patients with genomic data. The bar plots show the clinical course of the 43 patients with targeted sequencing and/or CNA data that passed QC for both their primary and metastatic samples, where stars indicate death. In the heatmap, green represents positive or high values (Age $>=50$, Size $>=2 \mathrm{~cm}$, positive node, Grade III, ER, PgR, HER2 positive, patient has a non-classic subtype, patient is de novo metastatic, patient has the treatment type, patient was processed for targeted sequencing, and copy number analysis), while grey represents the contrary and white the missing values.

Abbreviations: adj: adjuvant, chemo: chemotherapy, ER: estrogen receptor, HT: hormone-therapy, met: metastatic, PgR: progesterone receptor, prim: primary, QC: quality control, RT: radiotherapy, CNA: copy number aberration, sTIL, stromal tumor infiltrating lymphocytes.

Figure 3: Genomic landscape of the primary/metastatic matched samples from the EuroILC cohort. (A, C, D), green, red, blue in the heatmap represent alterations private to primary tumor, at least one private to the metastasis, and present in both the primary and the metastasis, respectively. Colors of the patient numbers refer to the histological type of the primary tumor of the patient with orange, turquoise, brown, purple referring to classic, mixed non-classic, solid and trabecular histological types respectively. (A) Mutational landscape from targeted sequencing approach 
concerning 32 patients. (B) Lollipop representations of the most altered genes in EuroILC cohort. Green, black and brown colors are for missense, truncating, in-frame mutations respectively. The Yaxis is the alteration frequency among patients. (C,D) Deletions and amplification respectively observed from the low pass whole genome sequencing on 21 patients. (E,F) Colors of the lines (E) and dots (F) refer to the histological type of the primary breast cancer tumor of the patient with same color code as above. (E) CIN score evolution from primary to matched metastatic sample in EuroILC. Median scores are taken when several samples are available per patient, increases and decreases are depicted in solid and dashed lines, respectively. (F) Differences in CIN scores between metastases and primaries against elapsing time between sampling of the primary and the metastasis. Black line depicts the linear regression between the two variables. (G) CIN scores distributions between primary and metastatic samples in green and red respectively across the different studied cohorts. There are 43, 41, 33, 94, 174, 361 patients available for EuroILC primaries, EuroILC metastases, MSK-IMPACT ILC primaries, MSK-IMPACT ILC metastases, MSK-IMPACT IDC primaries, MSK-IMPACT IDC metastases regarding copy number analyses, respectively.

Abbreviations: CIN: Chromosomal INstability, M: metastatic sample, P: primary sample.

Figure 4: Clinical course of 3 selected EuroILC patients. Aromatase inhibitors, ESR inhibitors, chemotherapy and radiotherapy are represented in dark green, green, red and dark red, respectively. Private alterations including mutations, short indels, copy number gains and losses are indicated under the corresponding processed samples. Samples available for sequencing analysis are shown in blue boxes.

Abbreviations: M: metastatic sample, Met: metastasis, P: primary sample.

Figure 5: Mutational landscape of ILC as compared to IDC in primaries and metastatic samples. (A-F) Percentage of patients having the alterations are shown on the axes. Level of significance is color coded according to the p-value adjusted for multiple testing using FDR, grey, pink, red for q-value<.01, <.1, >=.1 respectively. Genes differentially altered at least once between two series are labeled in all comparisons. ESRI and PIK3CA were not differentially altered but are 
labeled for information. There are 54, 40, 51, 135, 260, 563 patients available for EuroILC primaries, EuroILC metastases, MSK-IMPACT ILC primaries, MSK-IMPACT ILC metastases, MSK-IMPACT IDC primaries, MSK-IMPACT IDC metastases regarding mutation analyses, respectively.

Abbreviations: FDR: false discovery rate, IDC: invasive ductal carcinoma, ILC: invasive lobular breast cancer, M: metastatic, P: primary. 
Figure 1

A

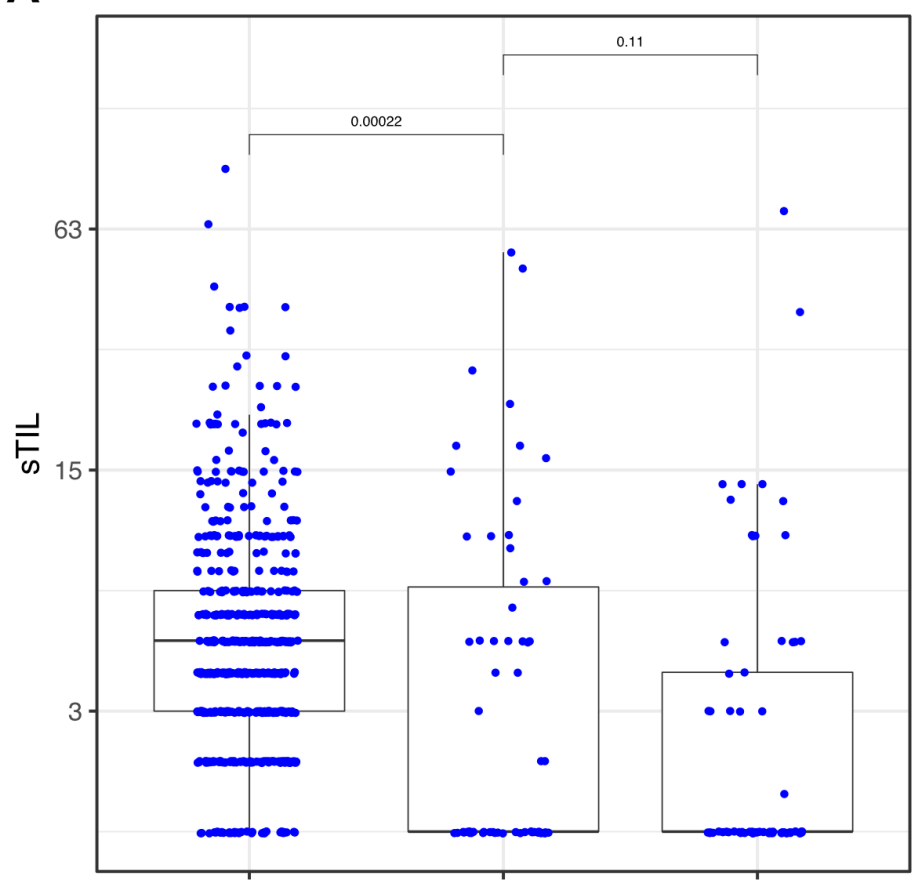

Prim(Desmedt 2018) Prim(EurolLC) Met(EurolLC)

C

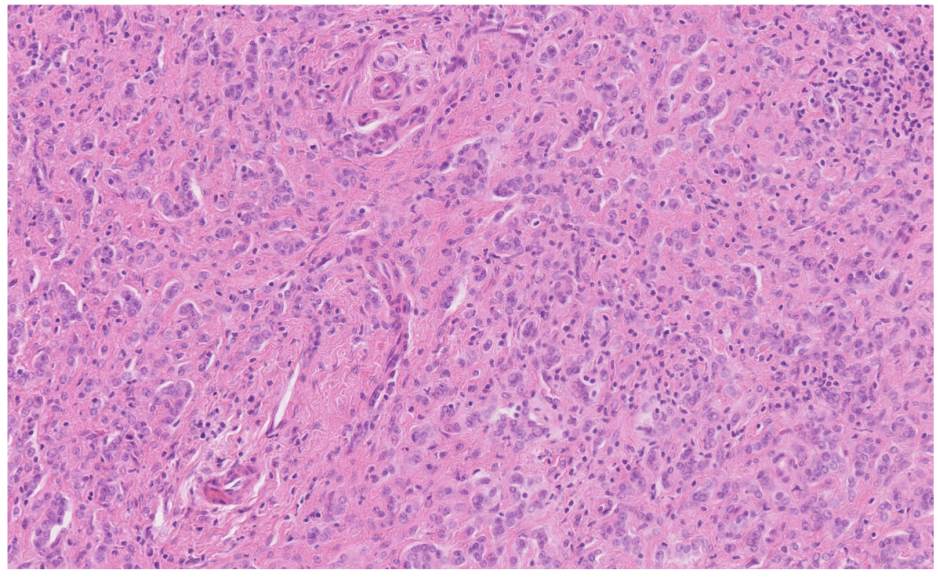

B

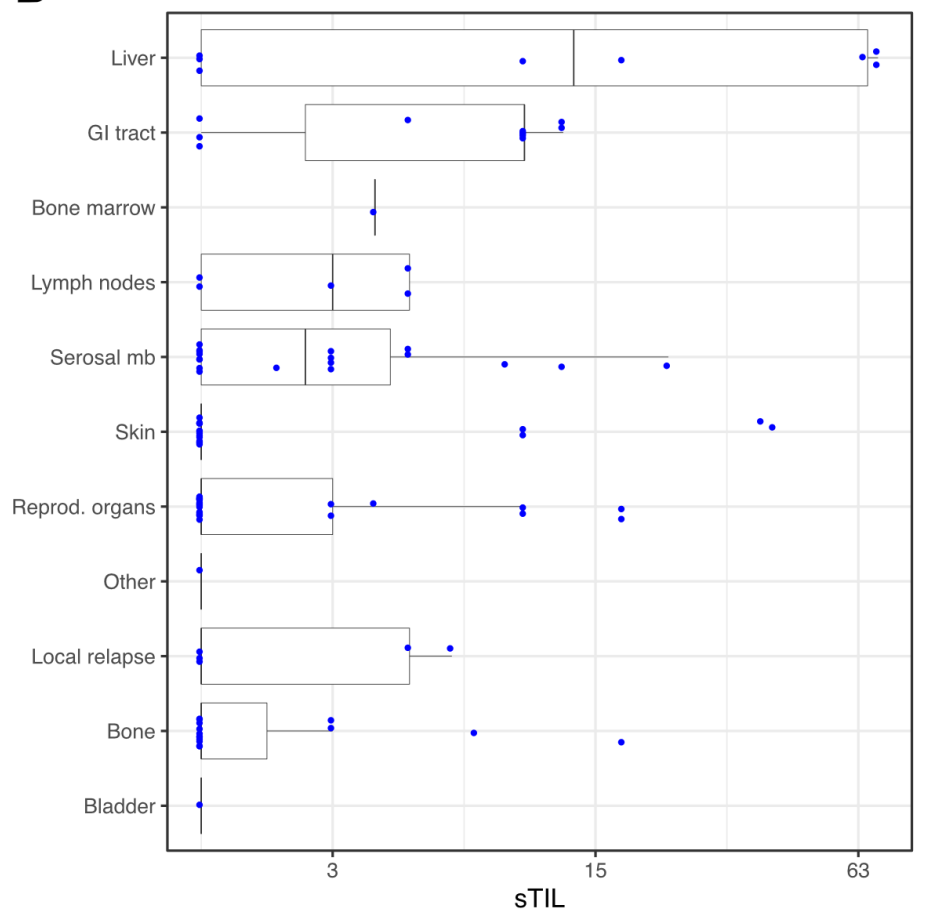

D

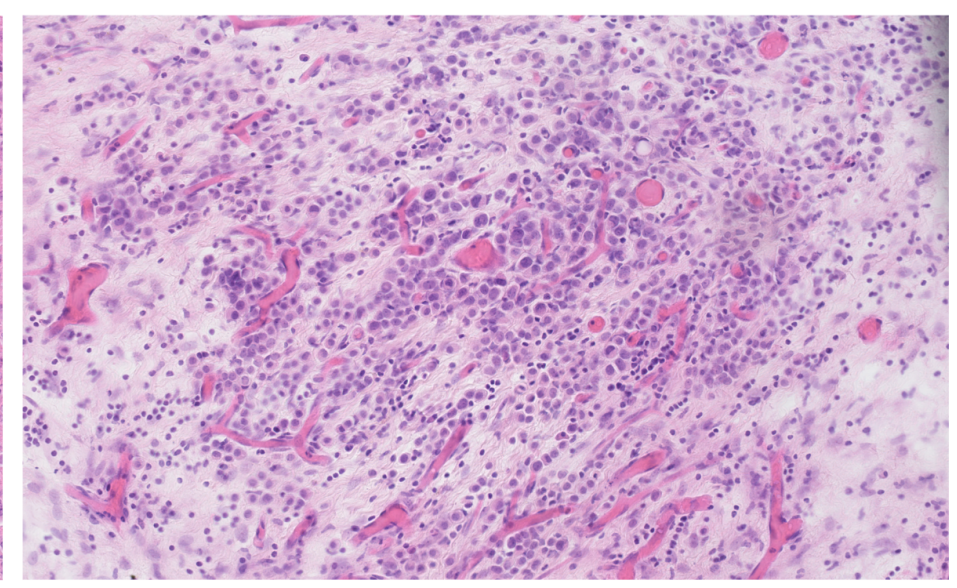


Figure 2

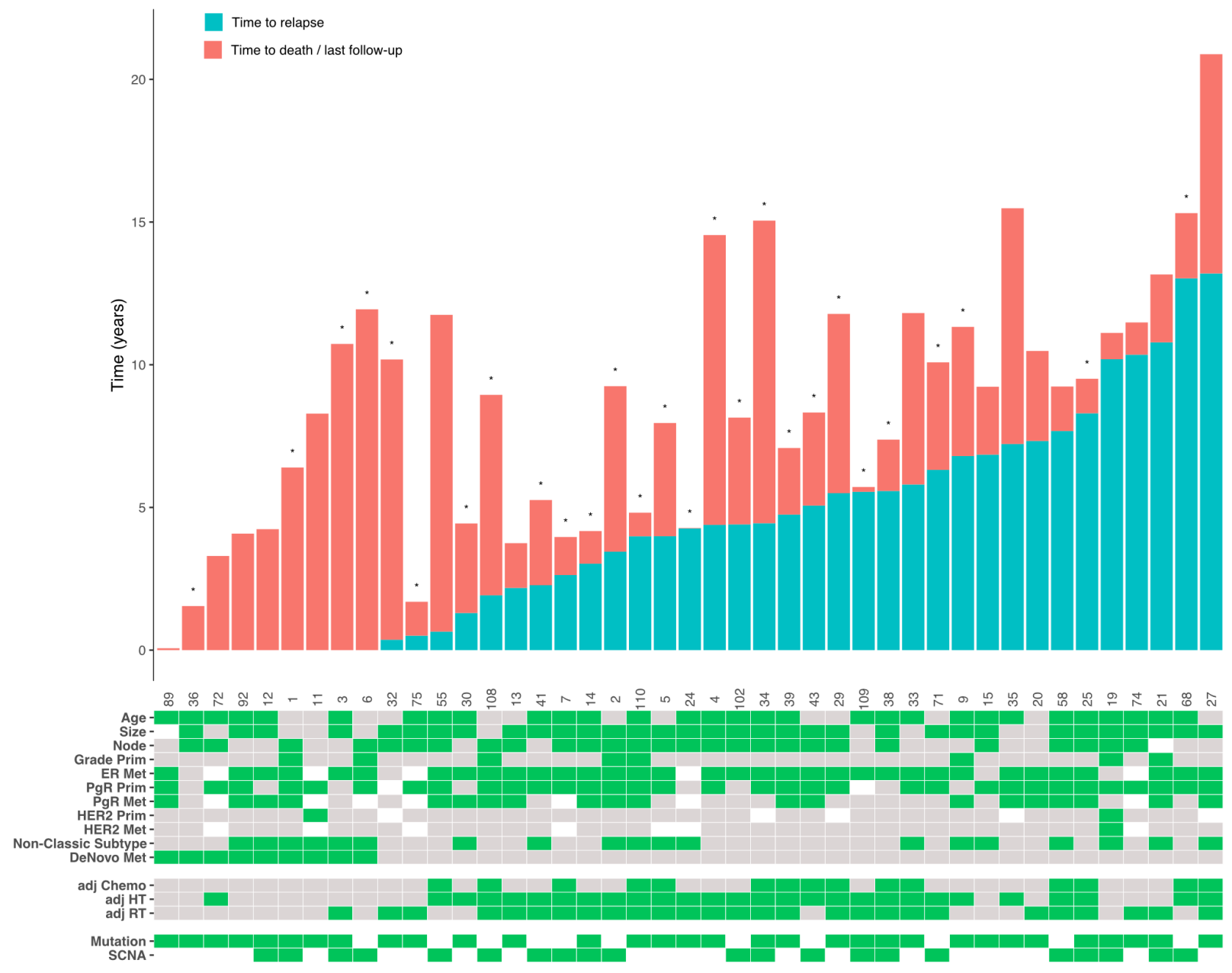




\section{Figure 3}

A

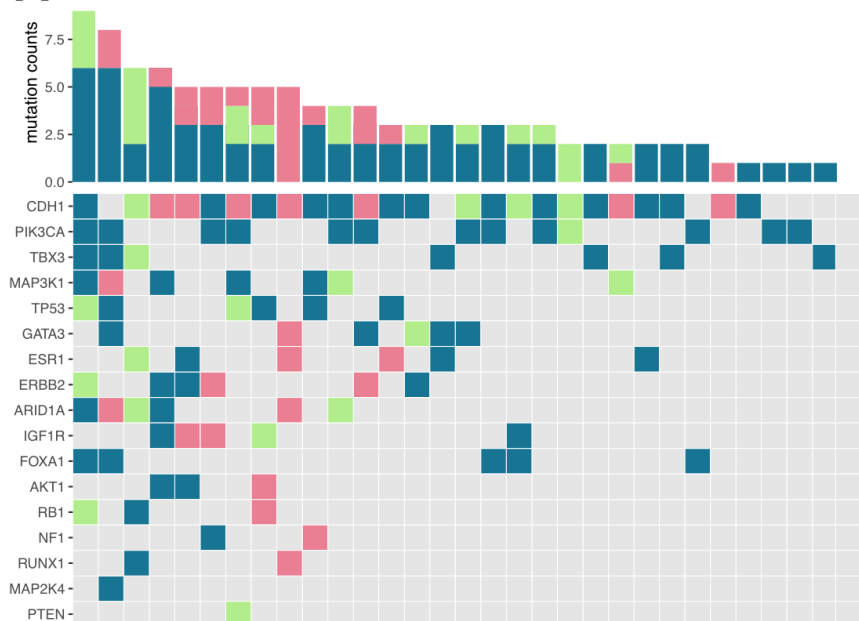

C
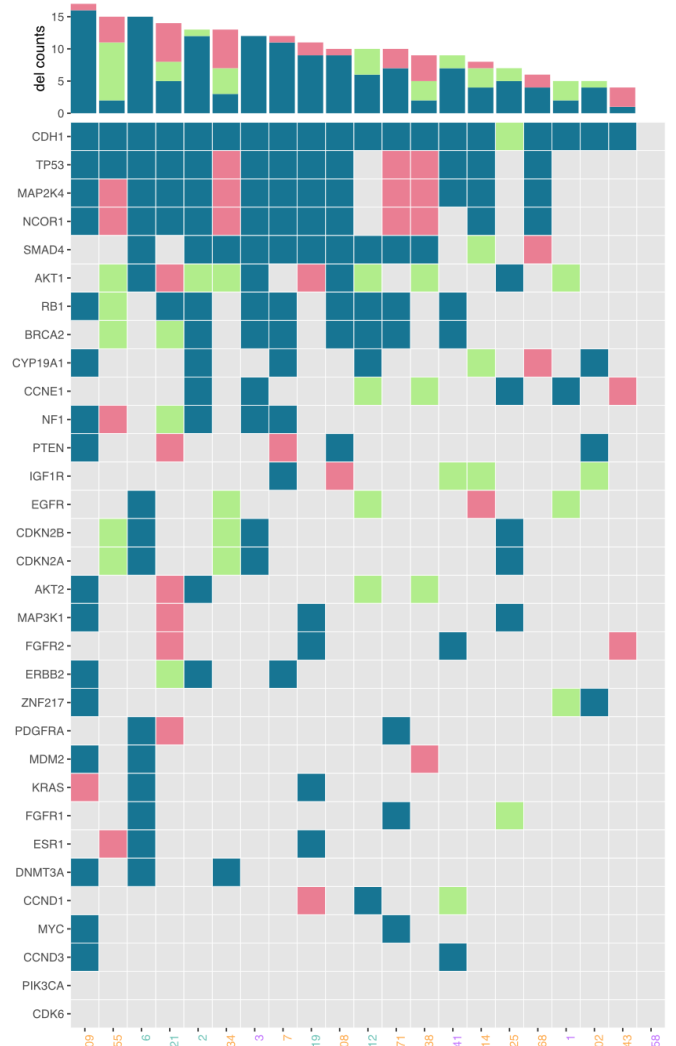

E

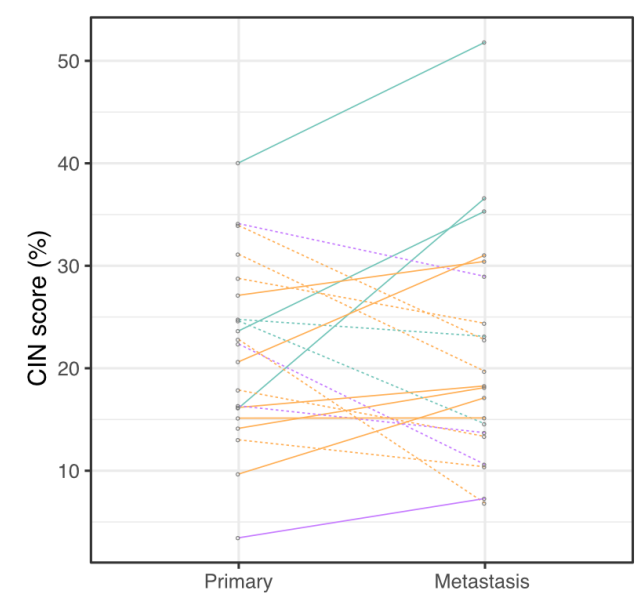

Alteration

Primary

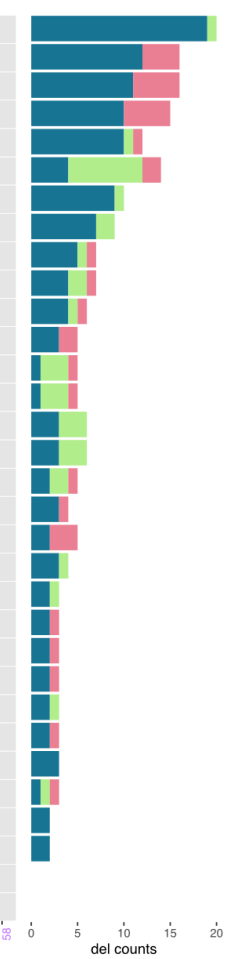

F

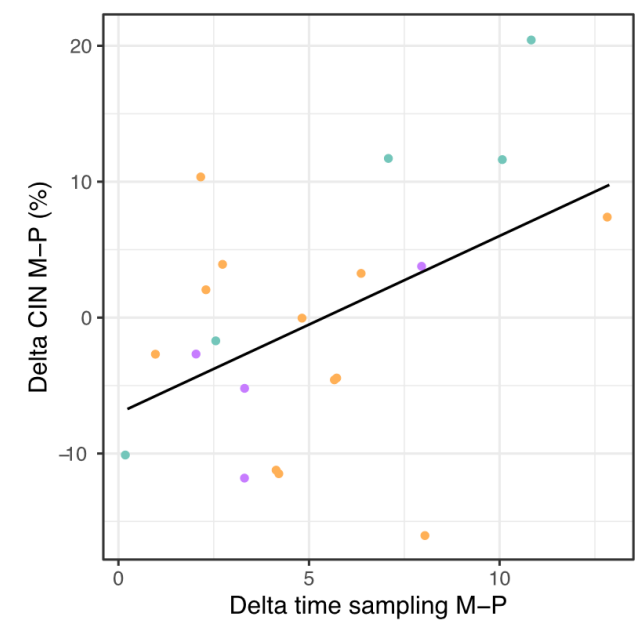

Subtype
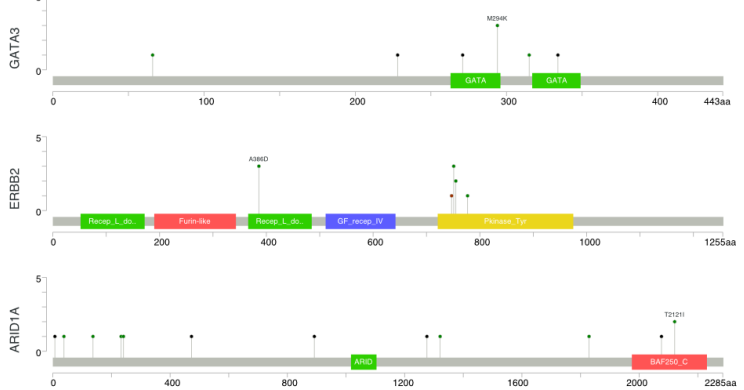

产

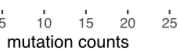

B

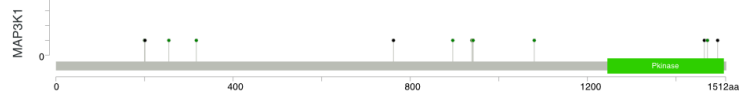

D

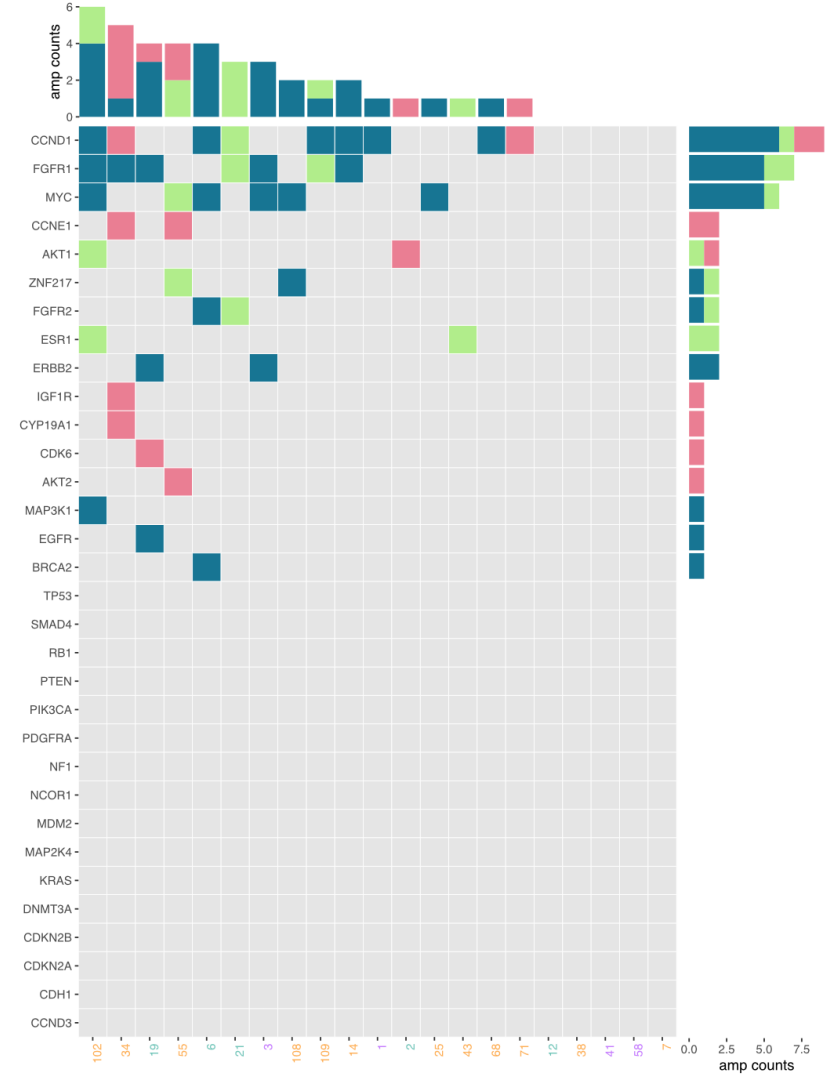

G

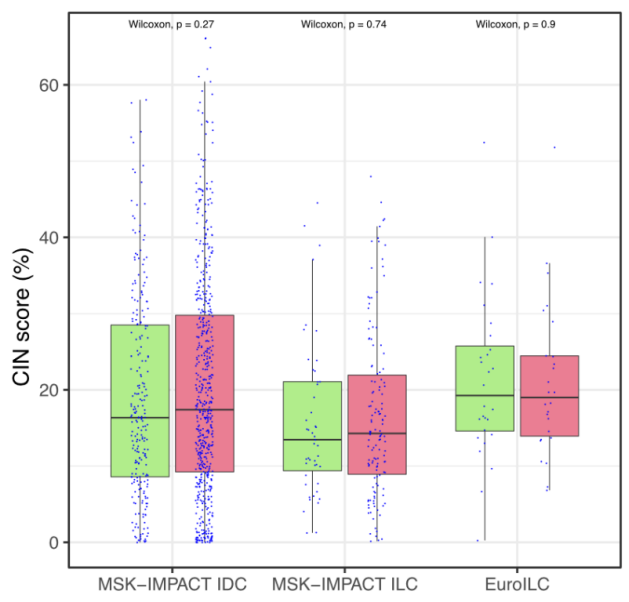


Figure 4

A

Patient 3

Postmenopausal

RFS: de novo

$1 \mathrm{y}$

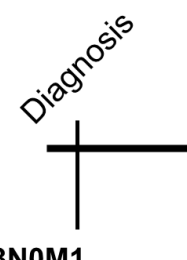

T3N0M1

Trabecular

ER+/HER2-

Ki67: 10\%

Met:lung

Treatments

Private alterations

Samples

B

Patient 34

Postmenopausal

RFS: $4.5 y$

$1 \mathrm{y}$

Treatments

Private alterations

mut: CDH1,PTEN,TP53

del: CDKN2A,CDKN2B,EGFR del: MAP2K4,NCOR1,TP53

amp: $\varnothing$

Samples

C

\section{Patient 21}

Postmenopausal

RFS: $10.8 y$

$1 \mathrm{y}$

Treatments

Private alterations

mut: $\varnothing$

del: $B R C A 2, E R B B 2, N F 1$

amp: CCND1,FGFR1,FGFR2

Samples

\section{P1}

Met:local relapse

ER+/HER2-

Ki67: 5\%

ER+/HER2missing

P2

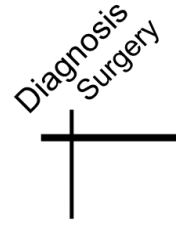

T4NmissingMo

Mixed non-classic

ER+/HER2-

Ki67: $40 \%$

$\{\varnothing\}$

$\{\varnothing\}$

P1

M1

P2

M2

P4

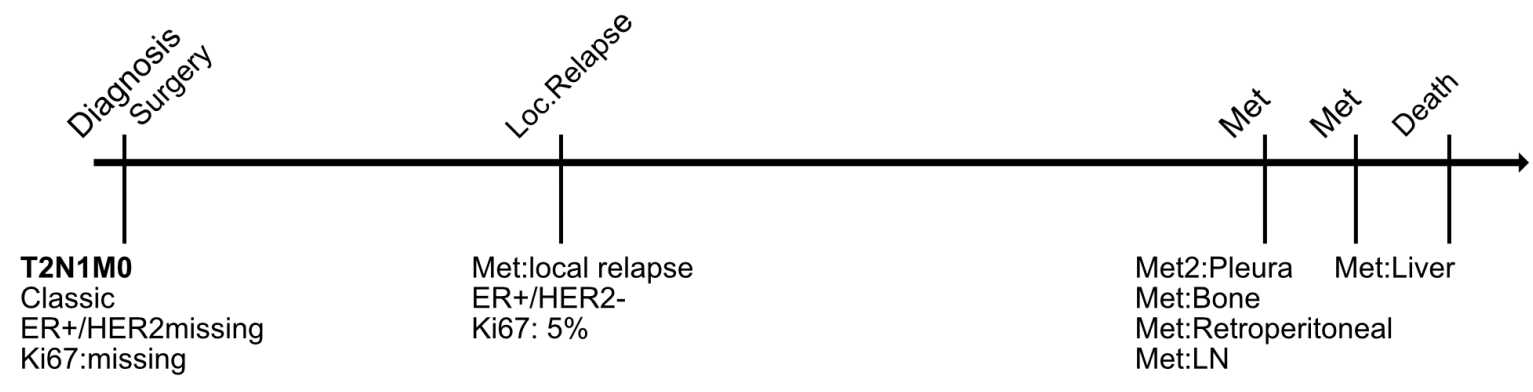

Met:skin-scar

ER+/HER2

Ki67: 15\% 
Figure 5

A

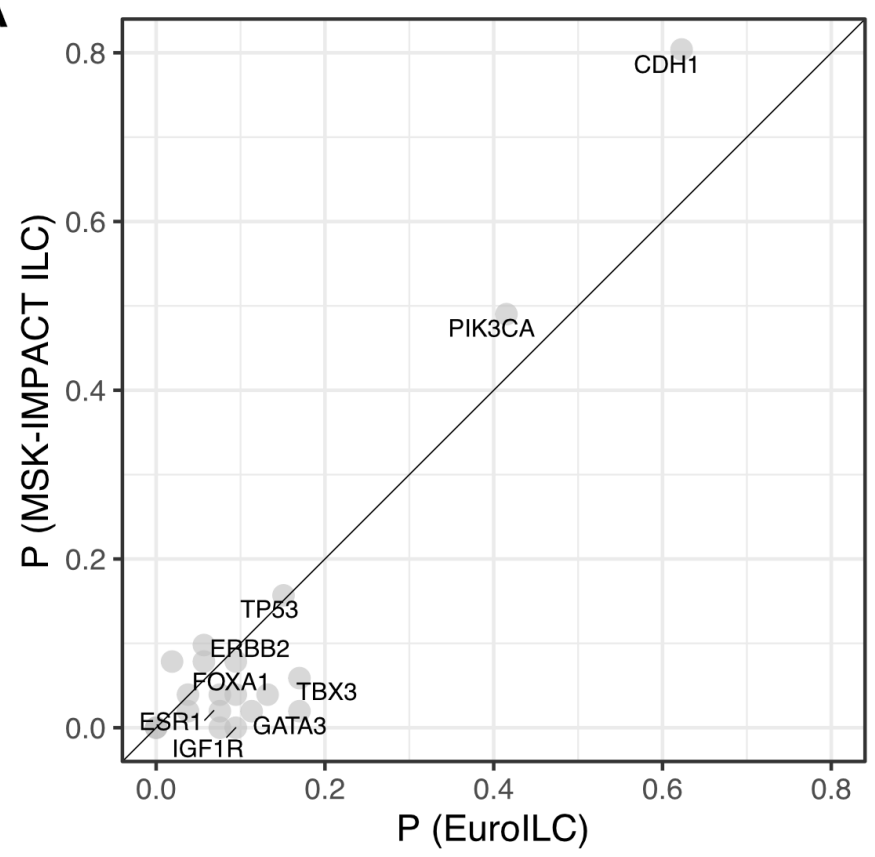

C

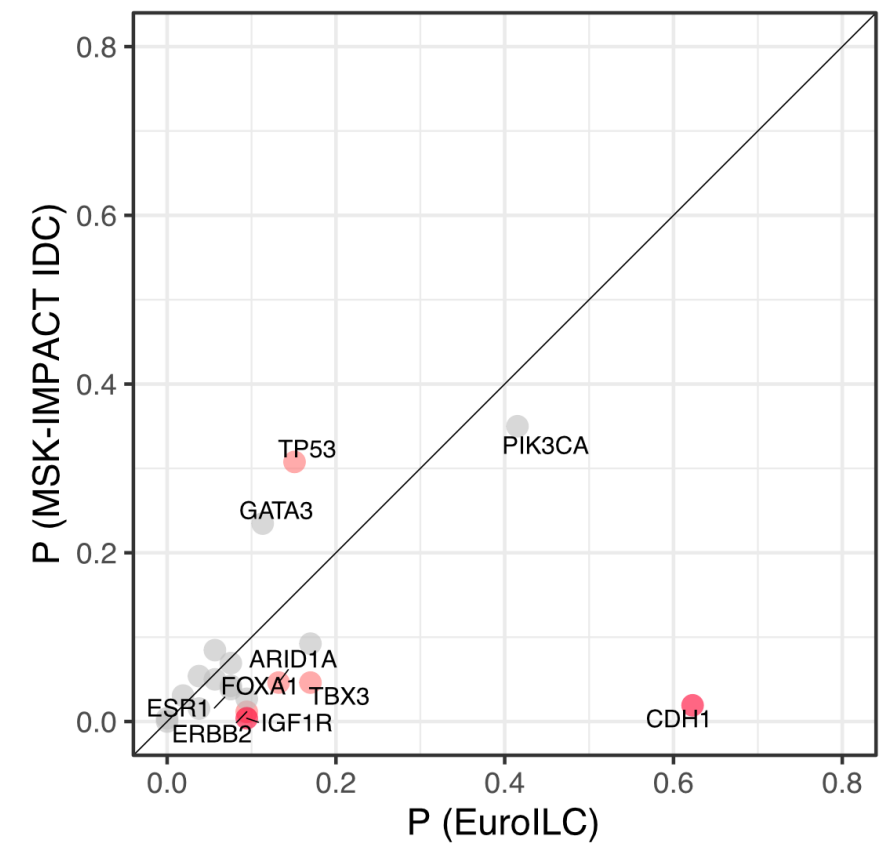

E

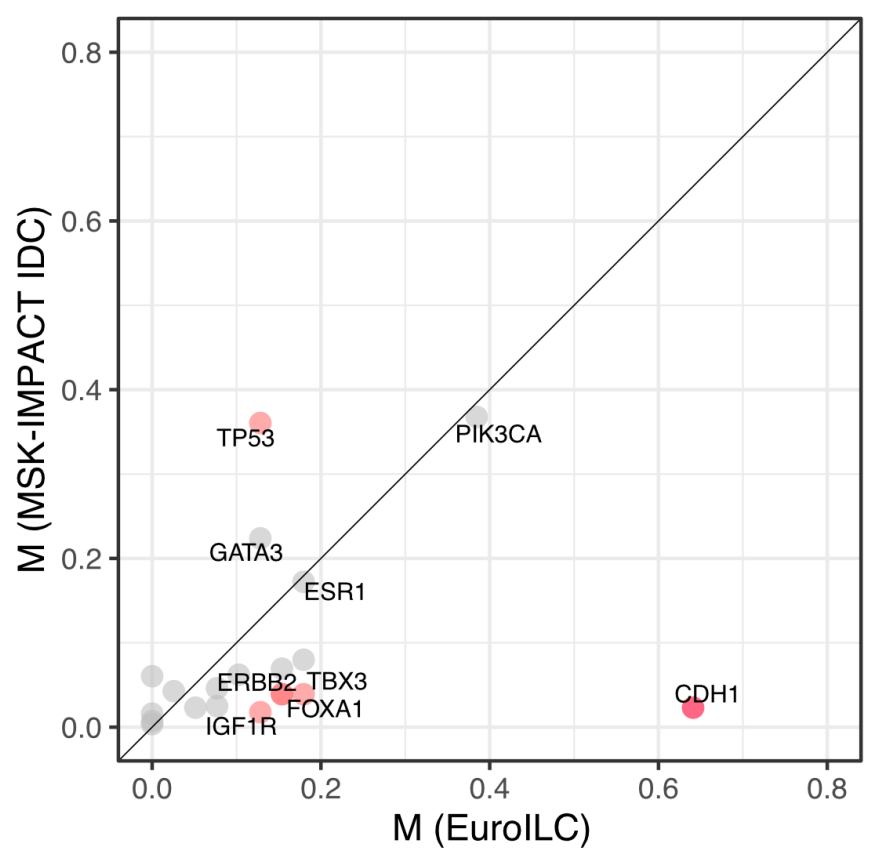

B

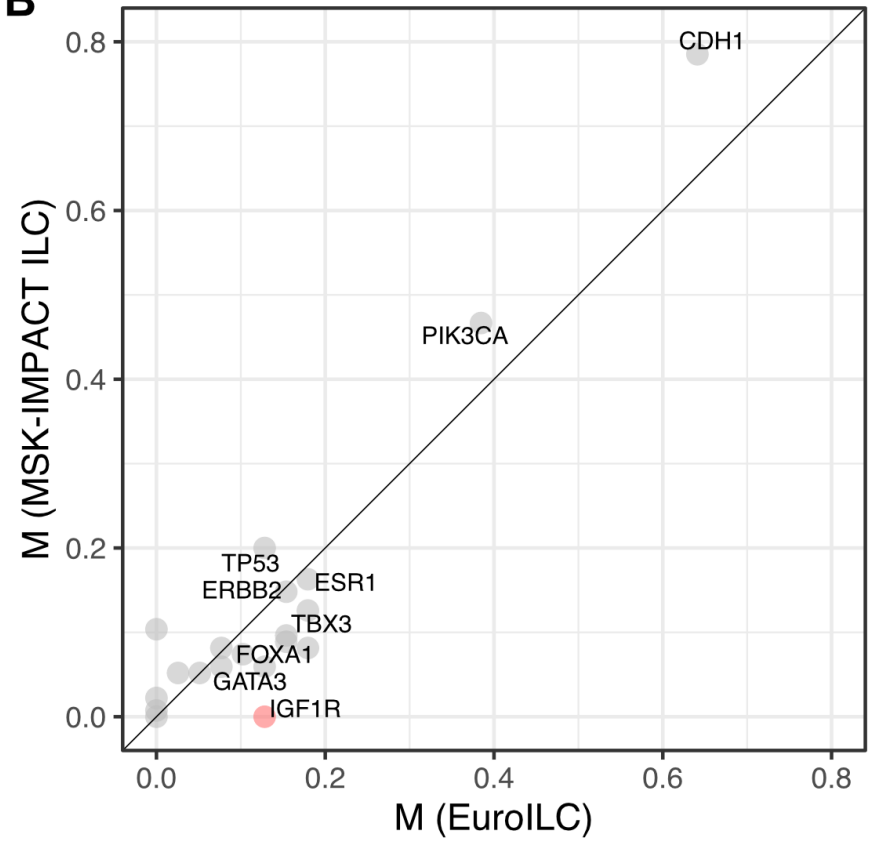

D

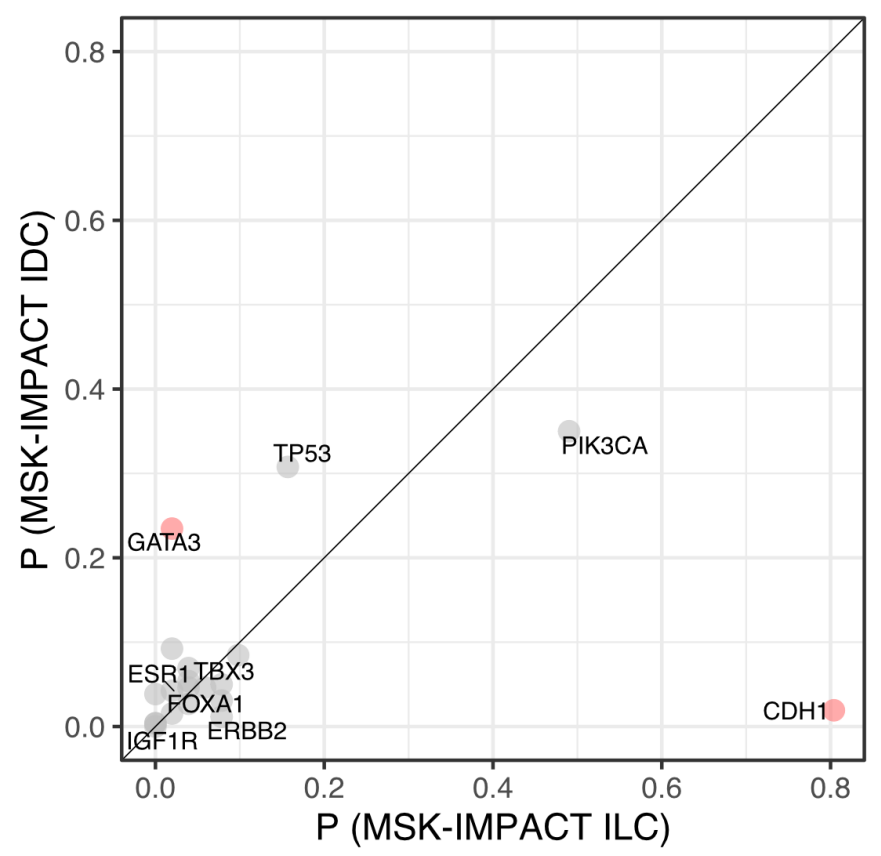

F

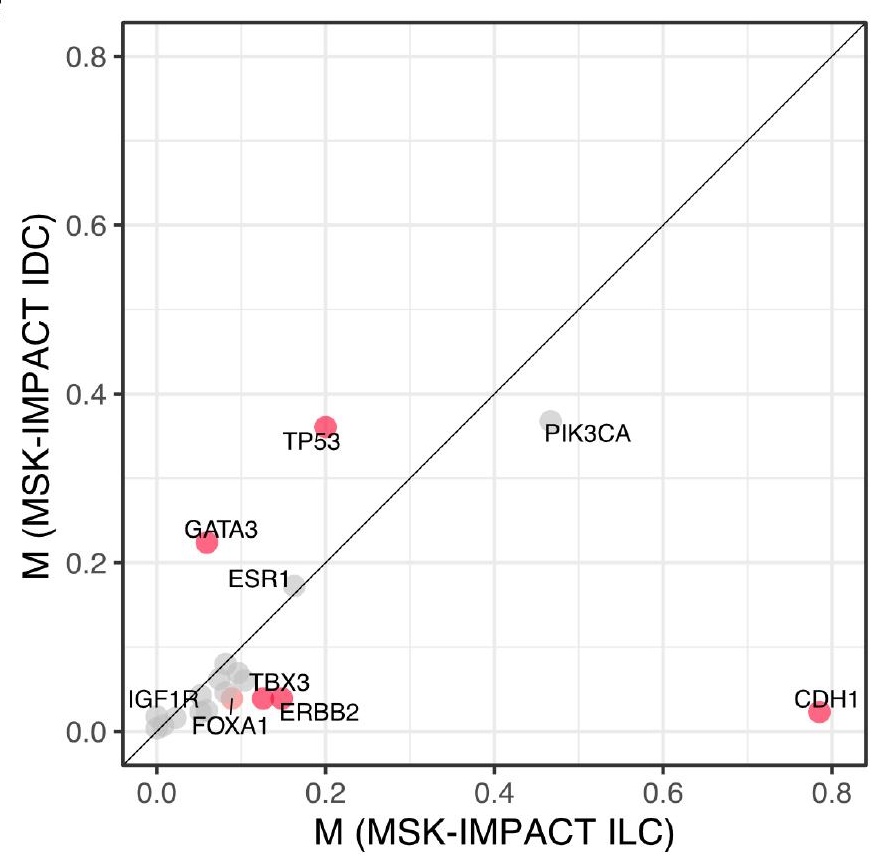

